\title{
Results from T2K
}

\author{
Ken Sakashita*t \\ HIGH ENERGY ACCELERATOR RESEARCH ORGANIZATION, KEK \\ E-mail: kenshepost.kek.jp
}

We report new results on electron neutrino appearance with the data collected until summer 2012. We observe 11 electron neutrino events with a background expectation of $3.22 \pm 0.43$ events. The probability to observe 11 or more events with a background-only hypothesis is $0.08 \%$, which corresponds to an equivalent significance of $3.2 \sigma$. We perform a fit for the observed $11 v_{e}$ candidate events using $p_{e}-\theta_{e}$ shape information and obtain $\sin ^{2} 2 \theta_{13}=0.094_{-0.040}^{+0.053}\left(0.116_{-0.049}^{+0.063}\right)$ for the normal (inverted) hierarchy and $\delta_{C P}=0$ assumption.

36th International Conference on High Energy Physics,

July 4-11, 2012

Melbourne, Australia

\footnotetext{
* Speaker.

${ }^{\dagger}$ On behalf of the T2K collaboration
} 


\section{Introduction}

The discovery of electron neutrino appearance in a muon-neutrino beam, $v_{\mu} \rightarrow v_{e}$, represents a direct detection of neutrino flavor mixing in appearance mode. This appearance mode plays an important role in the search for $\mathrm{CP}$ violation and the mass hierarchy because the oscillation probability of $v_{\mu} \rightarrow v_{e}$ depends on not only $\theta_{13}$ in the leading term but also CP violation and mass hierarchy. Therefore, $v_{\mu} \rightarrow v_{e}$ appearance can open the possibility to measure CP violation in the lepton sector. In 2011, we reported the first indication of electron neutrino appearance with the data collected until March 2011[1]. After our results, reactor experiments confirmed the non-zero value of $\theta_{13}$ through observation of electron-antineutrino disappearance 2, 3, 4. In this paper, we report updated results of electron neutrino appearance using the data collected by summer 2012 and improved analysis methods.

T2K (Tokai-to-Kamioka) [5] is a long-baseline neutrino oscillation experiment that uses an intense muon neutrino beam to measure the mixing angle $\theta_{13}$ via $v_{\mu} \rightarrow v_{e}$ appearance and the mixing angle $\theta_{23}$ and mass difference $\Delta m_{32}^{2}$ via $v_{\mu}$ disappearance. The muon neutrino beam is produced as the decay products of pions and kaons generated by the interaction of the $30 \mathrm{GeV}$ proton beam from J-PARC ${ }^{1}$ with a graphite target.

We employ the "off-axis" method[6] in order to produce the intense narrow-band neutrino beam. We set the angle to $2.5^{\circ}$ in order to tune the beam energy to be $0.6-0.7 \mathrm{GeV}$ which corresponds to the maximum of oscillation probability with $\Delta m_{32}^{2}=2.4 \times 10^{-3} \mathrm{eV}^{2}$ and the baseline of $295 \mathrm{~km}$. It is important to keep the beam direction stable because a shift of beam direction can cause a shift of the beam energy peak. We measure the beam direction with the secondary muon profile and successfully keep the beam direction within $1 \mathrm{mrad}$ (corresponding to a shift in the energy peak of less than $2 \%$ ).

We started physics data taking in January 2010. The data taking was stopped on March 11, 2011 by the Great East Japan Earthquake. After recovering most of the facilities and the recommissioning the beam, we resume the data taking in April 2012. The data taken from April to June 2012 includes runs with $200 \mathrm{kA}$ of horn current operation due to some failures of the horn power supply (while the nominal horn current is $250 \mathrm{kA}$ ). We analyze the full data collected: $3.01 \times 10^{20}$ POT, which is an $18 \%$ of increase over the results reported at Neutrino 2012 conference[7].

The properties of the neutrino beam are measured by the near detectors. We measure the neutrino interaction rate, beam direction and stability with the on-axis detector, INGRID. We measure the off-axis beam properties with the ND280 detector, which is positioned $2.5^{\circ}$ off-axis. It consists of two Fine Grained Detectors (FGD), three Time Projection Chambers (TPC), an Electromagnetic Calorimeter system (ECal), Side Muon Range Detectors (SMRD) and a $\pi^{0}$ detector (P0D). Except for the SMRD, the detectors are located inside of a dipole magnet, operated at $0.2 \mathrm{~T}$. We measure $v_{\mu}$ charged current (CC) events and use them in the oscillation analysis. We also measure the $v_{e}$ $\mathrm{CC}$ and $\mathrm{NC} \pi^{0}$ event rates in order to check the background events for $v_{e}$ appearance analysis.

The far detector (SK), located $295 \mathrm{~km}$ away from the target, is a water Cherenkov detector with a fiducial volume of $22.5 \mathrm{kton}$. We record all the PMT hits within $\pm 500 \mu \mathrm{s}$ from the beam arrival time, as determined by the GPS. The detector has good particle identification performance in the sub-GeV region for separating electron-like (showering) rings from muon-like rings. The

\footnotetext{
${ }^{1}$ Japan Proton Accelerator Research Complex
} 
probability that a muon will be mis-identified as an electron is $1 \%$, which we evaluate using atmospheric $v$ events.

\section{2. $v_{e}$ appearance analysis}

In $\mathrm{T} 2 \mathrm{~K}$, a signal event is a single electron-like ring event from a charged-current quasi-elastic (CCQE) interaction of the oscillated $v_{e}$. There are two main backgrounds. The first is NC1 $\pi^{0}$ production from $v_{\mu}$ and $\bar{v}_{\mu}$ in which $\pi^{0}$ decays to two photons; one of photons may be reconstructed as a $v_{e}$ candidate event if the two photons overlap or if one of them is not reconstructed. The second is intrinsic $v_{e}$ in the beam from $\mu$ or $K$ decays.

We perform the $v_{e}$ appearance analysis using not only event rate information but also event shape information of the $v_{e}$ candidate events. Moreover, we reduce uncertainty of the prediction at the far detector using the near detector information.

\subsection{Event selection}

In order to select $v_{e}$ candidate events, we require an event pass six selection cuts: (1) it must be fully contained in the far detector fiducial volume and within the T2K beam-timing window; (2) there must be one single electron-like ring; (3) it must have visible energy greater than $100 \mathrm{MeV}$; (4) there must be no decay electron candidate; (5) the two photon invariant mass (assuming the existence of a secondary ring) should be less than $105 \mathrm{MeV} / c^{2}$; (6) the reconstructed neutrino energy, using the lepton momentum vector and assuming a QE interaction, should be less than $1250 \mathrm{MeV}$. With those selection criteria, the efficiency of $v_{\mu} \rightarrow v_{e}$ signal is $60.7 \%$ while rejection efficiency for NC background and the intrinsic $v_{e}$ background is more than $99 \%$ and $80 \%$, respectively.

\subsection{Near-to-far extrapolation}

The number of $v_{e}$ candidate events and its shape distribution are described by product of the beam flux, oscillation probability, neutrino interaction cross section and detection efficiency at the far detector. In the analysis, we parameterize each component and evaluate those parameter uncertainties. The beam flux is parameterized by an energy-dependent normalization factor used to adjust the Monte Carlo (MC) predicted flux. We calculate the MC flux using hadron production data (primarily from CERN NA61) and the proton beam profile measurement. The normalization factor is evaluated using ND280 measurements with the knowledge of correlations between the ND280 and SK flux energy bins. The correlations are evaluated with the MC simulation and both external and in-situ measurements including data from NA61. We simulate neutrino interactions using NEUT[8] and its cross section model is also parameterized. Initial parameter values and the uncertainties are evaluated using external measurements.

We fit two-dimensional distributions of angle and momentum of the muon tracks for CCQE and non-QE enhanced samples of ND280 measurements to constrain flux and neutrino interaction cross section parameters. Figure 1 shows the muon momentum distributions of the CCQE enhanced sample with comparisons to the MC predictions before and after the fit. The fit results are extrapolated to the prediction at the far detector.

We summarize the systematic uncertainty on the predicted number of events at the far detector after using ND280 measurements in Table 1. The uncertainty coming from the beam flux and neutrino interaction cross section is reduced by almost half after using ND280 measurements. The 


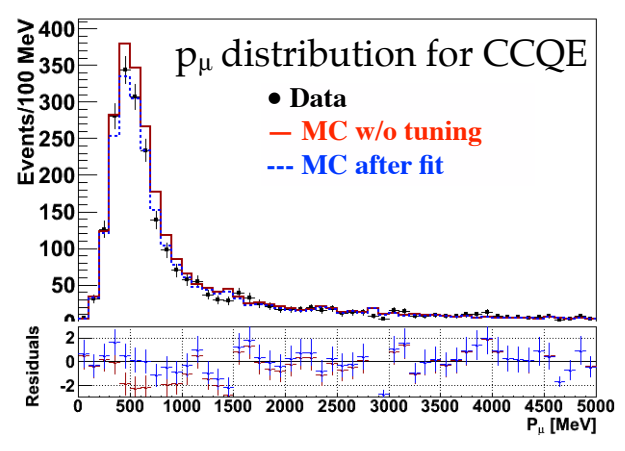

Figure 1: The muon momentum distribution of the ND280 CCQE enhanced sample. The MC distribution is shown as the red solid (blue dashed) histogram before (after) the ND280 fit.

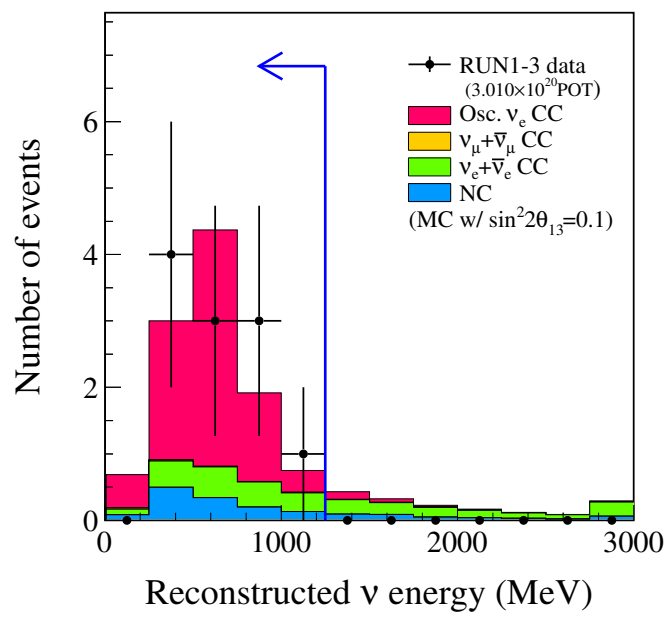

Figure 2: The reconstructed neutrino energy distribution with the signal and background expectations.

total systematic uncertainty on the predicted number of events for $\sin ^{2} 2 \theta_{13}=0(0.1)$ is $13.4 \%$ $(10.3 \%)$. We improve those systematic uncertainties compared to the T2K's 2011 results, which were $23 \%$ for $\sin ^{2} 2 \theta_{13}=0$ and $18 \%$ for $\sin ^{2} 2 \theta_{13}=0.1$, respectively. Table 2 summarizes the predicted number of events at the far detector for signal and background.

Table 1: Summary of the contributions to the error on the predicted number of events from each group of systematic sources.

\begin{tabular}{|c|c|c|c|c|c|}
\hline \multirow{2}{*}{ Error source } & \multicolumn{2}{|c|}{$\sin ^{2} 2 \theta_{13}=$} & \multirow{2}{*}{ Event category } & \multicolumn{2}{|c|}{$\sin ^{2} 2 \theta_{13}=$} \\
\hline & 0 & 0.1 & & 0 & 0.1 \\
\hline Beam flux $+v$ int. in $\mathrm{T} 2 \mathrm{~K}$ fit & $8.7 \%$ & $5.7 \%$ & Total & $3.22 \pm 0.43$ & $10.71 \pm 1.10$ \\
\hline$v$ int. (from other exp.) & $5.9 \%$ & $7.5 \%$ & $v_{e}$ signal & 0.18 & 7.79 \\
\hline Final state interaction & $3.1 \%$ & $2.4 \%$ & $v_{e}$ background & 1.67 & 1.56 \\
\hline Far detector & $7.1 \%$ & $3.1 \%$ & $v_{\mu}$ background & 1.21 & 1.21 \\
\hline Total & $13.4 \%$ & $10.3 \%$ & $\bar{v}_{\mu}+\bar{v}_{e}$ background & 0.16 & 0.16 \\
\hline
\end{tabular}

Table 2: The predicted number of events for each event category with POT $=3.010 \times 10^{20}$.

‥

\subsection{Oscillation analysis and results}

We apply the event selection for the data of $3.01 \times 10^{20}$ POT and observe $11 v_{e}$ candidate events. Figure 2 shows reconstructed energy distribution of the observed events. The probability to observe 11 or more events with a background-only hypothesis ( $3.22 \pm 0.43$ events) is $0.08 \%$, equivalent to an exclusion significance of $3.2 \sigma$. We find evidence of electron neutrino appearance.

We extract $\sin ^{2} 2 \theta_{13}$ from the observed $v_{e}$ candidate events using event rate and shape information. We perform a two-dimensional extended maximum likelihood fit to the $p_{e}-\theta_{e}$ distribution. We can obtain a better discrimination of signal events from backgrounds using the differences in 
the $p_{e} \theta_{e}$ distribution. Moreover, we perform two independent analyses; a maximum likelihood fit on the reconstructed energy distribution and a fit on only event rate information.

The likelihood is defined as the product of the likelihood for the observed number of $v_{e}$ candidate events $\left(\mathscr{L}_{\text {norm }}\right)$, the shape of the $p_{e}-\theta_{e}$ distribution of those events $\left(\mathscr{L}_{\text {shape }}\right)$ and a constraint likelihood term for the systematic uncertainties $\left(\mathscr{L}_{\text {syst }}\right)$. We use a Bayesian marginalization technique in order to incorporate the systematic uncertainties, by integrating over all systematic parameters.

$$
\mathscr{L}^{\prime}(\vec{o})=\int \mathscr{L}_{\text {norm }}(\vec{o}, \vec{f}) \times \mathscr{L}_{\text {shape }}(\vec{o}, \vec{f}) \times \mathscr{L}_{\text {syst }}(\vec{f}) d \vec{f} .
$$

where $\vec{o}$ is the set of oscillation parameters and $\vec{f}$ is the set of systematic parameters such as the beam flux normalization and neutrino cross section parameters. $\mathscr{L}_{\text {syst }}$ is the prior probability density function for the systematic parameters. We assume it to be a multivariate normal distribution with means and widths, and their correlations obtained from the near-to-far extrapolation. The oscillation parameters are obtained by maximizing the marginalized likelihood. The only variable oscillation parameter in the fit is $\theta_{13}$. We also extract the best fit value of $\theta_{13}$ with each value of $\delta_{C P}$ when we draw contours in $\sin ^{2} 2 \theta_{13}$ and $\delta_{C P}$ space ${ }^{2}$.

We perform a fit of the observed $11 v_{e}$ candidate events. Figure 3 shows the results of the fit assuming normal hierarchy and $\delta_{C P}=0$. The $68 \%$ and $90 \%$ C.L. intervals calculated using the Feldman \& Cousins 9 and constant $\Delta \chi^{2}$ method are found to be equivalent. The best fit value of $\sin ^{2} 2 \theta_{13}$ with its one standard deviation is $0.094_{-0.040}^{+0.053}\left(0.116_{-0.049}^{+0.063}\right)$ for the normal (inverted) hierarchy and $\delta_{C P}=0$ assumption. The $90 \%$ C.L. allowed region is $0.030<\sin ^{2} 2 \theta_{13}<0.175$ $\left(0.038<\sin ^{2} 2 \theta_{13}<0.212\right)$ for the normal (inverted) hierarchy case. Figure 4 shows the allowed region $68 \%$ (green) and $90 \%$ C.L. (blue) for $\sin ^{2} 2 \theta_{13}$ for each value of $\delta_{C P}$. The black solid line is the best fit value for each value of $\delta_{C P}$. This result is consistent with the fit on reconstructed energy distribution and the fit on the event rate information analysis.

\section{Summary and outlook}

We report new results on the $v_{e}$ appearance analysis based on $3.01 \times 10^{20}$ POT data (full data set collected up to summer 2012) which corresponds to $4 \%$ exposure of T2K goal. We observed $11 v_{e}$ candidate events at the far detector. The probability to observed 11 or more events with only background hypothesis ( $3.22 \pm 0.43$ events) is $0.08 \%$, equivalent to an exclusion significance of $3.2 \sigma$. We find evidence of electron neutrino appearance. We perform a fit of the observed $11 v_{e}$ candidate events using the $p_{e}-\theta_{e}$ shape information and obtain $\sin ^{2} 2 \theta_{13}=0.094_{-0.040}^{+0.053}\left(0.116_{-0.049}^{+0.063}\right)$ for the normal (inverted) hierarchy and $\delta_{C P}=0$ assumption. We plan to take more data with new runs at higher beam power toward a more precise measurement of $v_{e}$ appearance and aim to discover CP violation in the lepton sector.

\section{References}

[1] K. Abe et al. [T2K Collaboration], Phys. Rev. Lett. 107, 041801 (2011).

[2] Y. Abe et al. [DOUBLE-CHOOZ Collaboration], Phys. Rev. Lett. 108, 131801 (2012).

\footnotetext{
${ }^{2}$ Other parameters are fixed: $\Delta m_{21}^{2}=7.6 \times 10^{-5} \mathrm{eV}^{2}, \Delta m_{32}^{2}=2.4 \times 10^{-3} \mathrm{eV}^{2}, \sin ^{2} 2 \theta_{12}=0.8704, \sin ^{2} 2 \theta_{23}=1.0$, $\delta_{C P}=0$, travel length of $295 \mathrm{~km}$, earth matter density of $2.6 \mathrm{~g} / \mathrm{cm}^{3}$
} 

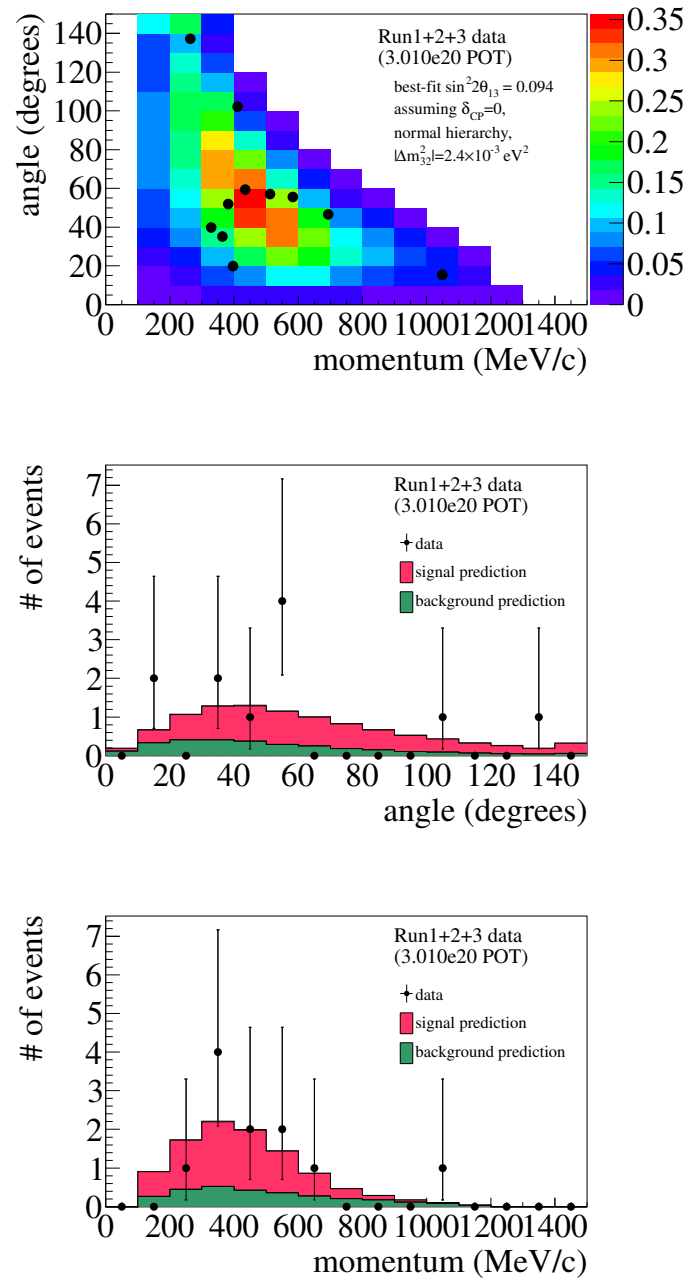

Figure 3: Top : the $p_{e}-\theta_{e}$ distribution of the $v_{e}$ events with the best fit value of $\sin ^{2} 2 \theta_{13}$ with the rate tuned from the near detector information overlaid with data (black dots) Middle : Same as above for the angular distribution. The expected signal (pink) and background (green) are shown in a stacked histogram. Bottom : Same as above for the momentum distribution
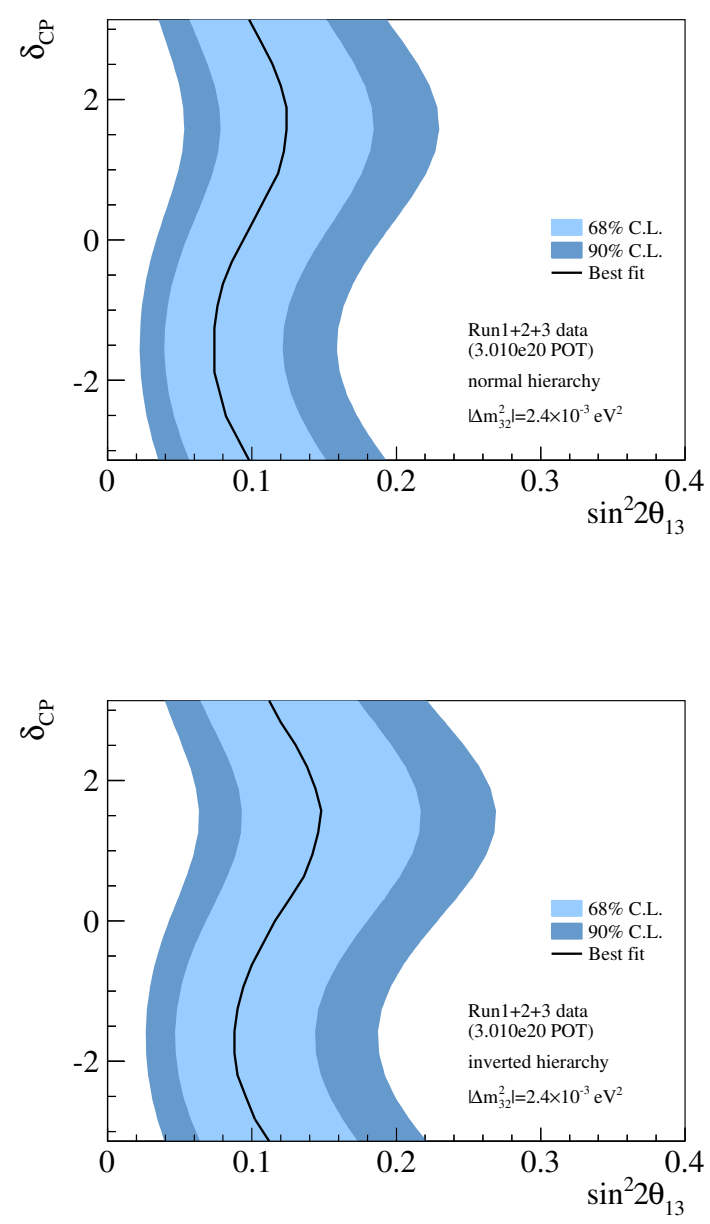

Figure 4: The $68 \%$ (green) and $90 \%$ C.L. (blue) allowed regions for $\delta_{C P}$ vs. $\sin ^{2} 2 \theta_{13}$ assuming the normal hierarchy (top) and inverted hierarchy (bottom) with all other oscillation parameters held fixed. The best fit for each value of $\delta_{C P}$ is also shown for the $p_{e}-\theta_{e}$ analysis (black)

[3] F. P. An et al. [DAYA-BAY Collaboration], Phys. Rev. Lett. 108, 171803 (2012).

[4] J. K. Ahn et al. [RENO Collaboration], Phys. Rev. Lett. 108, 191802 (2012).

[5] K. Abe et al. [T2K Collaboration], Nucl. Instrum. Meth. A 659, 106 (2011).

[6] D. Beavis, A. Carroll, I. Chiang, et al., Physics Design Report, BNL 52459 (1995).

[7] http://neu2012.kek.jp/

[8] Y. Hayato, Acta Phys. Polon. B 40, 2477 (2009).

[9] G. J. Feldman and R. D. Cousins, Phys. Rev. D 57, 3873 (1998). 